\title{
Mécanismes de Financement et Performance des IMFs en Contexte Camerounais: Une Application sur les COOPEC (Coopérative d'Epargne et de Crédit) et les IFNB (Institution Financière Non Bancaire)
}

\author{
Dadem Kemgou Edouard Guilaire (PhD) \\ Moniteur au Département de Finance-Comptabilité, Faculté des Sciences \\ Economiques et de Gestion, Université de Dschang, Cameroun \\ Wafo Hilaire Cabrel
}

Doctorant au Département de Finance-Comptabilité, Faculté des Sciences

Economiques et de Gestion, Université de Dschang, Cameroun

\section{Manetsa Eloge Lord}

étudiante Master II au Départent d'économie

et Sociologie Rural, Université de Dschang, Cameroun

doi: 10.19044/esj.2017.v13n19p332 URL:http://dx.doi.org/10.19044/esj.2017.v13n19p332

\begin{abstract}
The objective of this study is to identify how the policies of financing influence the performance of Microfinance institutions (MFI). In this study, we used the MIX Market database in order to bring out information relating to the variables of financing and performance structures. The data used was obtained from eight Institutions of Microfinance in Cameroon, and were collected over a period of eight years going from 2006 to 2013. An analysis of the regressions based on a sample of 64 observations collected on 8 Cameroonian MFIs for 8 years, on the one hand, shows that the level of indebtedness also influences financial and social performance negatively.
\end{abstract}

Keywords: structure of financing, social and financial performance, MFI

Résumé

Les activités d'octroi de Micro crédit ont été institutionnalisées dans le but de résoudre les problèmes de pauvreté qui prend une dimension congénitale. Dans le but de proposer des solutions afin de rendre pérenne ces institutions, cette présente étude a pour objectif de tester le lien entre la structure de financement des Institutions de Microfinance sur leurs performances aussi bien sociale que financière dans la sphère géographique du Cameroun (Nous avons retenu les IMF ayant comme statut légal 
COOPEC et IFNB). Une analyse des régressions sur un échantillon de 64 observations recueillir sur 8 IMF Camerounaise pendant 8 années, montre d'une part que le niveau d'endettement influence négativement aussi la performance financière que sociale.

Mots-clés: structure de financement, performance sociale et financière, IMF

\section{Introduction}

La marginalisation des plus pauvres du système bancaire classique a conduit à la mise en œuvre des institutions de Microfinance (IMF), dans un but d'apporter des services financiers aux acteurs du secteur informel (Ayayi et al., 2010). Dans cette démarche, l'expérience réussie de la Grameen Bank dans les années 80 a dynamisé la microfinance et impulsé une nouvelle façon d'appréhender la pauvreté. Malgré leurs faibles revenus, les exclus du système bancaire classique ont prouvés qu'ils peuvent honorer leurs engagements en termes de remboursement de prêts (à intérêt) et d'utiliser ces prêts de façon productive (Lapenu et al., 2007).

Cet engouement pour la Microfinance s'accompagne d'une augmentation de la demande des services auprès de ces mêmes institutions. En Afrique on compte pour 4,9 milliards de dollars, une clientèle d'environ 7,3 millions et un taux de croissance de clients de 10,9\% en 2012 (Mix market, 2012). Au Cameroun, les encours de crédit passeront de 135 milliards de FCFA en 2008 à 1953.5 milliards de FCFA en 2012 (MINFI, 2012).

Cette augmentation de l'offre des services de la Microfinance pose le problème de financement des institutions. Il est vrai que la Microfinance dispose d'une multitude de sources de financements (CGAP, 2007), cependant, une lecture comparative de l'architecture financière des IMF de par le monde, nous laisse constater une divergence selon que l'on soit dans des zones géographiques différentes (Mix market). En effet il ressort que les IMFs d'Afrique sub-saharienne se financent à hauteur de $72 \%$ en moyenne par l'épargne collectée. Toutefois, au Moyen Orient et en Afrique du Nord, la proportion de fonds propres est largement supérieure à celle de la dette.

Suivant un récent rapport (Mix market, 2013), l'Afrique subsaharienne est la région où les IMFs enregistrent le plus petit rendement compris entre $0 \%$ et $2 \%$. Ces constats deviennent de plus en plus préoccupants, quand on sait qu'une prise de décision efficace sur la structure du capital dans les IMFs, devrait minimiser les risques, optimiser la flexibilité financière tout en encourageant la solvabilité voulue à long terme qui devrait fournir un service financier durable aux clients pauvres (CGAP, 2007), il y a lieu de se poser la question suivante: quelles politiques les IMFs camerounaise en particulier et de l'Afrique sub-saharienne en générale 
devraient privilégier en termes de financement afin d'améliorer leurs performances tant sociale que financière? C'est sur cette question que va s'appesantir cette étude.

Cette problématique n'est pas nouvelle, car le débat sur la structure financière naît des travaux de Modigliani et Miller en 1958 de leur fameux article «the cost of capital, coporate finance, and theory of investment» dans lequel, ils soutiennent la thèse de la neutralité des sources de financements sur la valeur de l'entreprise. Ces travaux ont suscité des contestations qui les conduiront en 1963 à commettre un nouvel article où ils remettent en cause leur conclusion de 1958 dans des conditions d'existence d'une fiscalité des sociétés. En 1969 les travaux de Stiglitz qui introduisent le coût de faillite viennent remettre en cause les conclusions de 1963 selon laquelle il est préférable de financer son activité en totalité par la dette.

Une succession de théories expliquant l'existence d'une structure optimale de capital s'est construite. Parmi lesquelles les travaux d'Akerlof (1970) sur l'asymétrie d'information, Myers et Majluf (1984) sur la théorie du financement hiérarchique, Jensen et Meckling (1976) sur la théorie d'agence etc.

L'étude de cette problématique dans le contexte de la microfinance est rare, mais alors, on peut noter l'initiative des auteurs tels que Bogan et al. (2008), Coleman (2007). Toutefois ces travaux ne se sont pas intéressés à la sphère géographique de l'Afrique centrale encore moins dans le contexte camerounais. Une des particularités de cette étude est qu'elle s'intéresse à l'épargne, à la dette en générale ainsi qu'aux fonds propres.

L'objectif de cette étude est de mettre en évidence les mécanismes de financements et la performance des IMFs camerounaises. Cet objectif s'organisera en trois sous-objectifs:

Mettre en évidence l'influence de la dette sur la performance des microfinances camerounaises,

- Déterminer l'impact des fonds propres sur la performance des IMF au Cameroun,

- De présenter la place de l'épargne dans la définition de la performance au sein des IMF.

\section{La revue de la littérature}

\section{Revue de la littérature théorique}

La littérature nous enseigne qu'en théorie financière, on distingue trois grandes approches: l'approche classique, l'approche néoclassique et l'approche moderne. 


\section{Les approches classique et néoclassique de la théorie sur la structure financière}

L'approche traditionnelle de la théorie sur la structure financière supporte l'idée que l'entreprise a intérêt à s'endetter tant et aussi longtemps que la rentabilité économique est supérieure au coût de la dette (Vernimmen, 2002). Cette approche a pour élément central le concept d'effet de levier. En effet, pour les tenants de cette position, il existe un niveau de dette associé aux capitaux propres qui maximise la valeur de l'entreprise.

En 1958 les auteurs tels que Modigliani et Miller déclenchent une véritable révolution dans le monde académique et professionnel de la finance (Albouy et al., 2003, p. 65). Ces deux auteurs démontrent la non-pertinence de la structure du capital. En d'autres termes, "la valeur de la firme serait indépendante de son endettement, donc de sa politique de financement» (Albouy et al., 2003, p. 64). Suivant leurs argumentaires, le procédé d'arbitrage permet d'établir un équilibre sur le marché, de telle manière que la valeur totale d'une entreprise ne dépende que des estimations de l'investisseur quant aux risques et revenus attendus de la firme et non pas de sa structure financière. Très vite cette position doit être contestée, pour la raison selon laquelle les hypothèses de base sont irréalistes, un monde sans impôts ne pourra exister.

À la suite de cette critique, Modigliani et Miller introduisent le concept de la fiscalité corporative et concluent que la valeur de la firme devient proportionnelle à son niveau d'endettement. Si l'emprunt propose des conditions assez favorables à la firme qu'aux investisseurs, ceci, par le biais de la fiscalité, il est évident que l'entreprise endettée verra sa valeur augmenter par rapport à celle non endetté (Albouy et al., 2003, p.70). De ce fait, ils proposent qu'il soit optimal pour l'entreprise de se financer à 100\% par la dette. Ce qui parait une aberration du point de vue financier; car à chaque fois que l'entreprise emprunte pour financer son activité, elle compromet ses chances de survies.

Pour ce fait, Stiglitz (1976) émet l'idée qu'en présence de coût de faillite l'existence, d'une structure optimale de capital se confirme et représente la combinaison des capitaux propres et d'emprunt qui minimise ces coûts de faillite.

En plus de ces courants classique et néoclassique, une autre conception a vu le jour: celui de l'approche moderne sur la structure financière. Il s'agit d'une démarche visant à démontrer la pertinence de la structure de capital en évoquant des raisons autres que celle de la contestation des hypothèses de base du modèle de Modigliani et Miller (1958). 


\section{Théories modernes de la structure financière}

Considérant la relation entre un emprunteur et une banque, il est évident que l'emprunteur dispose des informations qui ne sont pas connues par la banque.

En effet, le taux d'intérêt est fixé et ne vari pas quelque soit le risque du projet, de manière à couvrir les risques de défaut des emprunteurs. Lorsque les banques font croitre les taux d'intérêt, cela peut conduire les emprunteurs à investir dans des projets dont les risques sont plus élevés que ceux prévus pour accroître leurs gains, ce qui conduira à augmenter le risque et à déprécier la valeur de l'entreprise.

La théorie de l'agence s'inscrit dans un contexte, où les recherches visent à apporter des raisons non fiscales afin d'expliquer le recours à l'endettement (Michel Albouy et al., 2003, p.240). Selon Jensen et Meckling (1976), la dette constitue le meilleur moyen de réduire les coûts liés aux manœuvres opportunistes du dirigeant. Ils démontrent que, l'incomplétude des contrats (problèmes d'asymétrie informationnelle) s'accompagne tout naturellement des coûts qu'ils nomment coûts d'agence. Selon Jensen (1986), ce conflit conduit généralement à la perte de valeur par l'entreprise. Ce risque, selon lui, existe dans des entreprises disposant des «Free Cash Flow». «Une telle situation implique un gaspillage et pour réduire les pertes de valeur, il est nécessaire de trouver les moyens obligeant les dirigeants à rendre ces Free Cash Flow aux investisseurs. Jensen suppose alors qu'un des moyens les plus efficaces de réduire les Free Cash Flow est le financement par l'endettement» (Albouy et al., 2003, p.134-135).

Selon Robinson (1997), la recherche de l'équilibre réside dans la sollicitation de l'épargne comme sources privilégiées de financement des institutions de Microfinance, car constitue une source de financement à moindre coût (Labie, 1999). C'est ce qui s'observe d'ailleurs dans les IMFs de l'Afrique subsaharienne où les proportions de l'épargne atteignent parfois plus de 70\% de l'actif (Mix market, 2012). La théorie de la répression financière trouve son essence dans les travaux de Mc Kinnon et Shaw en 1973. Ils présentent cette théorie comme la manifestation d'un certain nombre de restrictions entreprises par les pouvoirs publics. Au sens strict, il représente la fixation du taux d'intérêt débiteur en dessous du taux qui assure l'équilibre. Cette attitude liée à la baisse du taux créditeur, a pour effet de conduire les épargnants à s'abstenir quant à la constitution de l'épargne. Ceci entraîne chez les banques classiques, la raréfaction des fonds prêtables.

Dans cette circonstance ou la raréfaction des fonds est observée, il est inévitable pour ces institutions réglementées de pratiquer le rationnement du crédit. Les clients touchés n'ont pas de choix que de faire recours aux sources alternatives que proposent les IMFs. Ainsi ces intermédiaires financiers traditionnels verront l'épargne de ces clients marginalisés. Par ce mécanisme, la répression financière apparaît comme un vecteur du développement des 
institutions de Microfinance, donc de création de la valeur aussi bien sociale que financière.

\section{Revue de la littérature empirique}

Cela fait moins d'une décennie qu'on observe, des initiatives dans le domaine de la microfinance quant à l'influence de la structure du capital sur la performance. On peut citer comme celles de Bogan et al. (2007), Coleman (2007), et Haruna Sekabira (2013). Par ailleurs, d'autres travaux ont étudié l'impact de la structure du capital sur la performance des entreprises. C'est à ce sujet que Berger et Bonaccorsi dit Patti (2006) déclarèrent que le lien entre la performance et la structure du capital est assez étroit. Dans cette perspective, s'inscrit un ensemble de travaux parmi lesquels ceux évoqué précédemment qui stipulent que, un niveau élevé du ratio d'endettement ou un niveau bas de fonds propres réduit les couts d'agence, en incitant le manager à s'intéresser à l'intérêt des actionnaires. Plusieurs travaux ont confirmés ces résultats à l'instar d'Abor (2005). Par contre, d'autres études ont carrément contredit les conclusions précédemment évoquées. On peut citer Abor (2007) qui démontre que la structure du capital (dettes à long terme et le total des dettes) est négativement liée à la performance financière. Abor (2007) trouve une relation négative entre la structure du capital et la performance (Maina \& Mwasa, 2014; Abdul, 2012).

\section{Impact du financement sur la performance financière}

En Afrique, Europe de l'Est, Amérique du Sud, Moyen orient et Asie du sud, au cours d'une période allant de 2003 à 2006, Bogan et al en 2008 montrent que le financement par les subventions a un impact négatif sur la performance financière des institutions de la microfinance. L'auteur sollicite comme mesure de la performance financière, l'autosuffisance opérationnelle et financière (OSS et FSS). A la suite de ces résultats, il suggère que les subventions réduisent la performance financière du fait $\mathrm{du}$ manque d'incitation des propriétaires et des dirigeants qui n'ont pas personnellement investi leur fonds au sein de l'institution.

Au regard de ce qui a été dit jusqu'ici sur la dette, on peut affirmer d'un point de vue théorique que l'impact de la dette sur la performance financière est incertain. Très peu de travaux ont étudié le lien entre la structure du capital et la performance dans les IMFs. Coleman (2007) montre que les ratios des dettes à court termes et le montant total de dette rapportés à l'actif total ont un effet positif sur la réduction du taux de défaut par conséquent sur la performance financière de l'institution. Coleman réalise son étude sur 52 IMFs Ghanéenne sur une période de 10 ans (1995 à 2004). Il retient comme variables dépendantes, le rendement des actifs d'une part et d'autre part, le rendement des fonds propres. Comme variables indépendante, 
il considère: le total des dettes, les dettes à court terme, les dettes à long terme.

Silva (2008) à la suite de Coleman (2007), trouve que les ratios du total de la dette et des dettes à court terme ont un impact significatif et positif sur le ROE tandis que l'effet sur le ROA est significatif et négatif. En même temps, le ratio de la dette à long terme à un impact positif et significatif sur le ROE pourtant non significatif sur le ROA. Ces travaux soulignent le fait que les dettes à long terme ne contraignent pas le dirigeant de quelques façons que ce soient d'être attentif dans la gestion de l'IMF. En effet, l'auteur effectue ses recherches sur un échantillon de 290 IMFs issues de 61 Pays. Les indicateurs de performance retenus sont le ROE et le ROA. De même, il utilise un ensemble d'indicateur de la structure du capital à savoir: le total des dettes, dette à court terme, dette à long terme rapportées aux fonds propres d'une part, et d'autre part ces mêmes variables rapportés à l'actif total.

\section{Impact du financement sur la performance sociale}

La performance sociale s'observe suivant plusieurs horizons, les deux principales sont: la largeur et la profondeur de la portée. Les travaux de Bogan et al. (2008), nient tout effet de la structure de financement sur la performance sociale de l'IMF. Selon la conclusion de son étude, les sources de financements à savoir: la dette, les dépôts ou même les subventions, n'ont pas d'effet significatif sur la performance sociale.

Les conclusions des travaux de Bogan (2008) son battu en brèche par les travaux de Coleman (2007). Selon ce dernier, la dette influence positivement sur la largeur de la performance sociale mesurée ici par la rotation annuelle des clients. A la suite de cette conclusion, on serait poussé à considérer qu'afin de réduire le risque de défaut, les IMFs sont amenées à diversifier leurs clientèles.

En 2010, Amendariz et al ont démontré que l'incertitude des subventions aurait des effets pervers sur l'action de réduction de la pauvreté; ce qui pourrait par conséquent conduire à une dérive de mission. En effet, ils montrent que l'incertitude des subventions fait croitre la dérive de mission. En suite, ils testent sur un échantillon de 230 institutions de Microfinances originaire de 60 pays et pris sur une période de 8 ans (1999-2006), l'effet des subventions sur la réduction de la pauvreté. Leurs résultats montrent que les subventions ont un effet positif sur la profondeur de l'action de l'IMF donc sur sa performance sociale. Dans le même temps, les auteurs montrent que, l'incertitude des subventions a pour action de faire croitre le taux d'intérêt des prêts et par là induit une corrélation négative avec la portée de l'action de l'IMF. Développement des hypothèses 
Le nécessaire changement d'échelle qui permettra de servir plus largement la masse du public cible repose sur les tendances actuelles d'implication des banques commerciales et le renforcement de l'investissement privé (Littlefield \& Rosenberg, 2008). C'est dans cette dynamique que sont construits deux courants de pensées dans les réflexions autour des préoccupations sur la microfinance. Il s'agit des welfaristes et des institutionnalistes. Si ces deux écoles ont un objectif commun; la réduction de la pauvreté, ils divergent complètement sur la façon d'y parvenir. Les welfaristes insistent sur la portée sociale et l'analyse d'impact tandis que, les institutionnalistes s'intéressent à la clientèle (ménages pauvres) et à la pérennité financière de l'institution (Adair \& Berguiga, 2010).

Pendant que les uns nient son influence sur la performance (Modigliani et Miller, 1958), d'autres démontrent son implication dans la création de la valeur (Jensen, 1986; Grossman \& Hart, 1982). Même lorsque certains auteurs sont d'accord sur le fait qu'elle influence la valeur de l'entreprise, ils divergent sur son effet. Grossman et Hart (1982) affirment que, les gestionnaires dont le moyen principal de financement est par fonds propres, n'ont aucune raison incitative à maximiser le profit de la firme.

Le dirigeant peut être tenté d'engager des investissements destructeurs de valeur, en présence de Free Cash Flow (Jensen, 1986). Jensen et Meckling (1976) nous enseignent que la relation qui lie le gérant et les actionnaires est entachée des coûts qu'ils nomment coûts d'agence. Ces coûts ont pour effets de contribuer à l'inefficience de l'organisation dans sa globalité. Pour remédier à cela, ils proposent un financement par la dette. Bonaccorsi et al. (2006) affirment qu'un niveau élevé du ratio d'endettement (un niveau bas du ratio des fonds propres) sur l'actif total, réduit les coûts d'agences et augmente la valeur de la firme.

Toutefois, lorsque le ratio d'endettement devient relativement élevé, promouvoir son augmentation générera un coût d'agence non négligeable qui elle-même entrainera l'augmentation du coût de faillite et la naissance d'un conflit entre les préteurs et les actionnaires (Berger \& Bonaccorsi-di patti, 2006). Un argument, tout à fait logique, considère que la dette peut réduire le pouvoir discrétionnaire des dirigeants sur les ressources de l'entreprise et par conséquent leur capacité à faire face aux pressions concurrentielles (Simerly \& $\mathrm{Li}, 2000)$. Le niveau d'endettement pourrait ainsi affecter des projets nouveaux et risqués, tels que la $\mathrm{R} \& \mathrm{D}$, souvent déterminants pour la survie à long terme de l'entreprise (Kochhar, 1996).

Il ressort de ce qui précède une divergence d'opinions sur l'apport de la dette à la performance de l'entreprise. Suite à l'ensemble des arguments avancés, nous pouvons déduire l'hypothèse $\mathrm{H}_{1}$ ci-dessous; 


\section{$\mathrm{H}_{1}$ : la dette affecte positivement la performance financière et négativement la performance sociale des IMFs.}

En ce qui concerne les IMFs, le débat sur la part de responsabilité des dépôts sur la performance de l'institution n'est pas traité avec un réel engouement, puisque très peu de travaux les indexent de façon directe, elle a presque toujours été traitée comme une variable de contrôle. Pourtant, en 2003, la Micro Rate \& Inter American Développent Bank, soulignait déjà ce que les IMF effectuant très souvent les opérations de collecte d'épargne ont un coût de fonds relativement bas, ce qui inéluctablement favorisera la création de la valeur (contribuera à rendre performante l'institution).

La situation qui se présente est que l'épargne qu'elle mobilise aura pour origine les populations démunies donc les faibles épargnants. Or suivant une étude faite par la CGAP et la Fondation Grameen, les très petites épargnes ne sont presque pas rentables (CGAP, 2007). C'est pourquoi en décidant d'avoir le cœur net sur le réel impact qu'a l'épargne sur la performance des l'IMFs au Cameroun, nous orientons un pan de notre recherche à mettre en évidence la relation qui pourrait exister entre les dépôts et la performance. D'où l'hypothèse 2 (H2) suivante:

\section{$\mathrm{H}_{2}$ : l'épargne influence négativement la performance financière et positivement la performance sociale.}

Les welfairistes tel que: Caroll (1979) et Servet (2007) estiment que, le point de départ du raisonnement welfairistes se fonde dans la théorie de la responsabilité sociale. Ils prônent une offre de services financiers à des coûts relativement bas. Ils encouragent ainsi un recours aux subventions. Le débat sur les fonds de subvention et leurs apports à la performance s'est intensifié dans le contexte des IMFs. Amendariz et al. (2010) affirment que, les subventions aident les IMFs à atteindre les pauvres dont d'assurer leur objectif social. On peut alors dire que les subventions contribuent à l'efficience sociale, ou plutôt à l'efficience financière des IMFs bien qu'elle soit marginale (Nawaz, 2010). Un niveau faible de subvention a pour conséquence l'augmentation des taux d'intérêt des prêts accordés par l'IMF à leurs clients (Caudil et al., 2009).

Mais ces résultats sont nuancés par Hudon et Traça (2011) qui affirment que, les subventions ont un impact positif sur l'efficience jusqu'à une certaine limite au-delà de laquelle, l'effet marginal devient négatif. Si le volume et la fréquence d'octroi des subventions aux IMFs étaient clairement spécifiés, les subventions allaient avoir un impact très important dans l'effort de réduction de la pauvreté par les IMFs. En plus des subventions, les IMFs reçoivent aussi des agences de développement des dons. Ils constituent pour la Microfinance un instrument financier afin d'améliorer leur capacité de pouvoir contracter des crédits auprès des banques à des coûts relativement 
bas en garantissant un taux de solvabilité élevé (Counts, 2005). Les subventions, les dons tout comme les actions constituent dans la microfinance ce qui est appelé fonds propres. Les défenseurs de la dette comme source de financement efficace militent pour un niveau de capitaux propres le moindre possible (Grossman et al., 1982; Jensen \& Meckling, $1976 \ldots$...). Par contre Simerly et Li en 2000 signifient le caractère primordial des capitaux propres. Ce développement nous permet de formuler l'hypothèse $\mathrm{H}_{3}$ ci-après:

$\mathrm{H}_{3}$ : les fonds propres influencent positivement la performance financière et négativement la performance sociale

\section{L'approche Méthodologie Présentation de l'échantillon}

Les données utilisées dans cette étude sont issues de la basse de données de la «Microfinance Informations Exchange» (MIX) disponible en ligne sur le site www.mixmarket.org. Il s'agit d'une base de données secondaire. En effet, le MIX garanti la transparence d'informations de plus de 1900 IMFs de part le monde. Jusqu'en 2012, 30 microfinances camerounaises de première et deuxième catégories émettent des informations sur le MIX. Notre échantillon est constitué de huit (8) Microfinances Camerounaises avec données recueillies sur huit (8) ans entre 2006 et 2013. Le caractère restreint de notre échantillon émane d'une indisponibilité d'informations dans notre base de données.

Tableau $N^{\circ} 1$. Caractéristique de l'échantillon

\begin{tabular}{|c|c|c|}
\hline \multicolumn{1}{|c|}{ L'institution } & $\begin{array}{c}\text { Statut } \\
\text { légal }\end{array}$ & $\begin{array}{l}\text { Nombre de périodes (2006 à } \\
\text { 2013) }\end{array}$ \\
\hline CCA (Crédit Communautaire de Croissance) & IFNB & $\mathbf{8}$ \\
\hline ACEP Cameroun & IFNB & $\mathbf{8}$ \\
\hline $\begin{array}{c}\text { RENAPROV (Reserve Nationale de l'Epargne } \\
\text { et de la Provision) }\end{array}$ & IFNB & $\mathbf{8}$ \\
\hline ADVANS Cameroun & IFNB & $\mathbf{8}$ \\
\hline CDS (Crédit Du Sahel) & IFNB & $\mathbf{8}$ \\
\hline $\begin{array}{l}\text { SOFINA (société Financière Africaine) } \\
\text { Coopératives) }\end{array}$ & IFNB & $\mathbf{8}$ \\
\hline MC2 (Mutuelle Communautaire de Croissance) & CoopEC & $\mathbf{8}$ \\
\hline
\end{tabular}

Source: l'auteur

\section{Les variables de la recherche}

\section{Mesure de la performance financière}

Le rendement des fonds propres (ROE) a été retenu dans nos travaux comme mesure de la performance financière en raison de l'exclusion, au niveau du numérateur, des éléments hors exploitation (tels que les subventions). Ce ratio est fréquemment utilisé pour mesurer la rentabilité 
commerciale. En général, ce ratio de rendement des fonds propres est calculé net d'impôts sur le résultat. Les IMFs qui n'utilisent pas le montant moyen des capitaux propres pour le dénominateur doivent préciser la nature du dénominateur utilisé (CGAP, 2003).

Nous retenons dans la littérature plusieurs travaux qui se sont servis de cette grandeur. On peut citer les travaux de Kar (2012) et Silva (2008).

\section{Mesure de la performance sociale}

L'hypothèse relative au montant moyen des prêts est que, plus le montant moyen du prêt par emprunteur est faible, plus l'IMF sert les pauvres. Mersland et Strom (2009), Ayayi et Sene (2010), de même que Crombrugghe et al. (2008) puis Hartarska (2005) utilisent dans leurs travaux le solde moyen de prêt par emprunteur. A la différence de ces auteurs nous allons utiliser la valeur du logarithme népérien Ln (AL) afin de réduire les écarts entre les valeurs pour éviter les biais.

Pour mesurer l'étendue ou la largeur de la portée des services offerts par les IMFs, Mersland et Strom (2009) ainsi que Luzzi et Weber (2006) utilisent le nombre des clients. Tout comme dans les travaux de Hartarska et Nadolnyak (2007) et Hartarska (2005) à la différence que ces deux auteurs, utilisent le logarithme du nombre des emprunteurs actifs afin de mettre en évidence la largeur de la portée.

\section{Variables liées à la structure de financement}

La littérature présente un ensemble de variables caractéristiques de la structure de financement. Abor $(2005,2007)$, Coleman (2007) utilisent la Dette à long terme, la dette à court terme et le total dette en pourcentage des actifs. Bogan et al. (2008) quant à eux utilisent les subventions en pourcentage des actifs. Dans notre étude, nous utiliserons six (6) variables de la panoplie des variables existante. Il s'agit en fait de:

- $\quad$ Le ratio des dettes totales sur l'actif total (DTA).

- $\quad$ Le ratio des dettes à court terme sur l'actif total (DCTA).

- $\quad$ Le ratio des dettes à long terme sur l'actif total (DLTA).

- $\quad$ Le ratio de dépôts total sur l'actif total (ETA).

- $\quad$ Le ratio des fonds propres sur le total actif (FPA).

- $\quad$ la variable subvention sur fonds propres (SUBFP).

\section{Les variables de contrôles}

A la suite des variables dépendantes, nous inclurions des variables de contrôles. Il en existe une variété; cependant, nous avons porté le choix sur deux facteurs principaux: les facteurs que nous avons retenus ont été utilisés par Trong Vi Ngo dans ses travaux de thèse en 2012. Néanmoins dans nos 
travaux, nous avons remplacé la variable proportion de prêt accorder aux femmes par la variable pourcentage de femmes emprunteuse afin de mieux cerner la considération accordée aux femmes dans la microfinance. Pour ce fait, nous travaillerons donc avec le pourcentage de femmes emprunteuse (PFE), l'échelle des opérations (ln(GLP)).

\section{Cadre conceptuel}

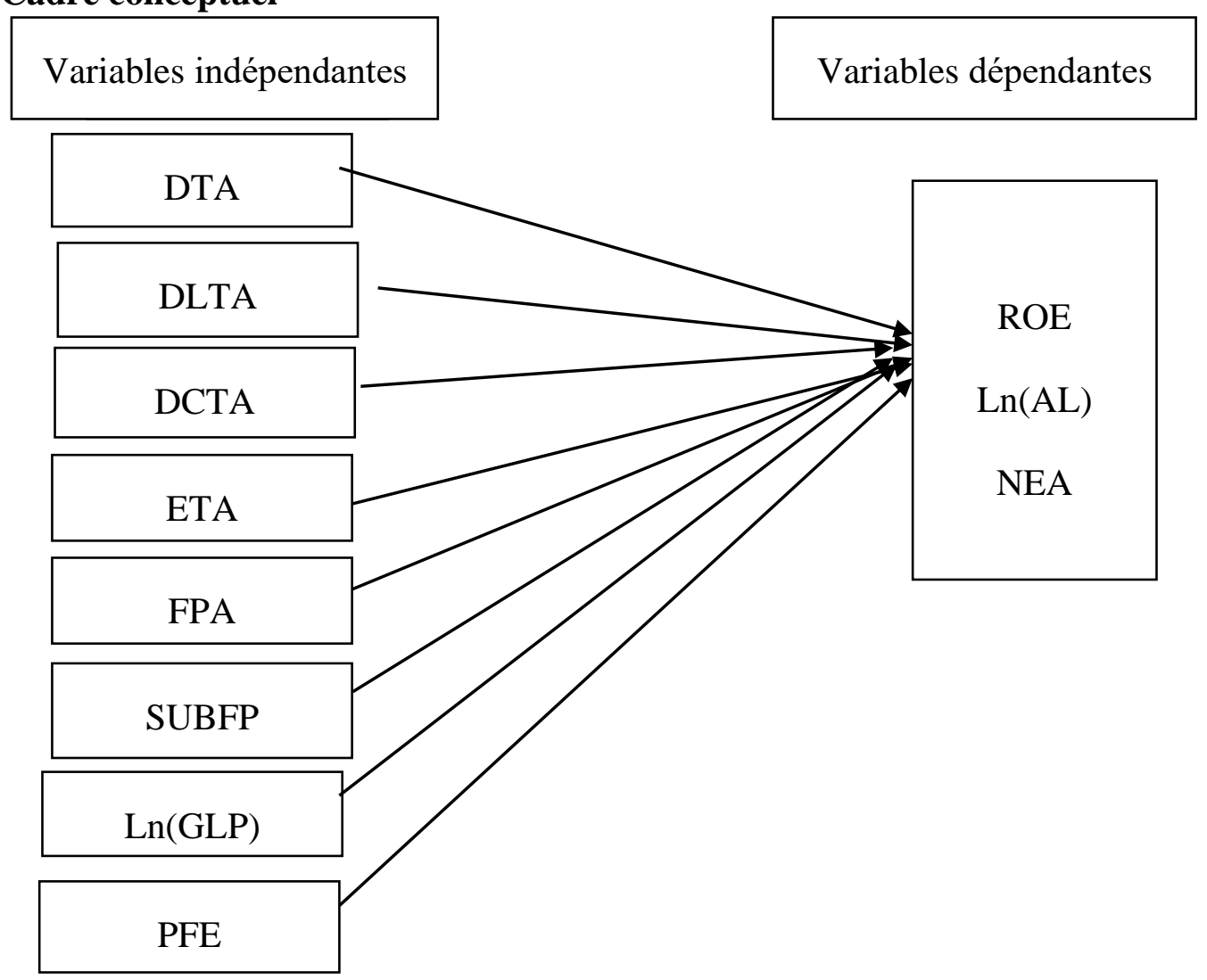

DLTA présent dans le modèle 1 et absent dans le modèle 2

ETA présent dans le modèle 2 et absent dans le modèle 1

Source: auteur à partir de la littérature

\section{Le modèle}

Le modèle qui sera retenu dans notre étude aura pour objectif de mesurer l'impact des sources de financement (fonds propres, dettes, épargne) sur la performance des IMFs. On traitera de la performance financière et de la performance sociale. Nos variables s'expriment dans le temps et dans l'espace. Au regard de la nature de nos données, nous avons opté pour un modèle de panels, car, c'est le seul qui permet de prendre en compte cette double dimension des données. C'est dans ce contexte que Kyereboah- 
Coleman (2007) et Bogan et al. (2008) ont réalisés avec succès leurs études sur l'impact de la structure du capital sur la performance de l'IMF, il s'écrit:

$$
\mathbf{Y}_{\mathrm{it}}=\mathbf{a}_{0 \mathrm{i}}+\mathbf{a}^{\prime} \mathbf{x}_{\mathrm{it}}+\boldsymbol{\varepsilon}_{\mathrm{it}}
$$

$\mathrm{i}=1 \ldots 8$ (les IMFs Camerounaise);

$\mathrm{t}=1, \ldots, 8$ (les années de 2006 à 2013) ;

Yit: la performance de la microfinance i observée à la date $t(R O E \operatorname{Ln}(A L)$ $\mathrm{NE}$ );

$\mathrm{X}^{\prime}{ }_{\mathrm{it}}$ : vecteur des $\mathrm{K}$ variables exogènes (variables explicatives).

$\mathrm{X}^{\prime}{ }_{\mathrm{it}}=\left(\mathrm{x} 1 \mathrm{it}, \mathrm{x}_{2 \mathrm{it}}, \ldots, \mathrm{xkit}\right)$;

a0i: terme constant (la constante) pour la microfinance $\mathrm{i}$;

$\mathrm{a}^{\prime} \mathrm{i}$ : vecteur des $\mathrm{K}$ coefficients des $\mathrm{K}$ variables explicatives.

$\mathrm{a}^{\prime}{ }_{\mathrm{i}}=(\mathrm{a} 1 \mathrm{i}, \mathrm{a} 2 \mathrm{i}, \ldots, \mathrm{aki})$;

Eit: le terme d'erreur.

Les formes empiriques de nos modèles s'écrivent:

Performance $_{i t}=\mathrm{a}_{0 \mathrm{i}}+\mathrm{a}_{1}$ DTA $_{\text {it }}+\mathrm{a}_{2}$ DLTA $_{\text {it }}+\mathrm{a}_{3}$ DCTA $_{\text {it }}+\mathrm{a}_{4} \mathrm{FPA}_{\text {it }}+$

${ }_{a_{5} S_{S U B F P}}+a_{\text {a }} \mathrm{PFE}_{\mathrm{it}}+\mathrm{a}_{7} \mathrm{Ln}(\mathrm{GLP})_{\mathrm{it}}$

$+\varepsilon_{\text {it }}$

Performance $_{i t}=\mathrm{a}_{0} \mathrm{i}+\mathrm{a}_{1} \mathrm{DTA}_{\mathrm{it}}+\mathrm{a}_{2} \mathrm{DCTA}_{\mathrm{it}}+\mathrm{a}_{3} \mathrm{ETA}_{\mathrm{it}}+\mathrm{a}_{4} \mathrm{FPA}_{\mathrm{it}}+\mathrm{a}_{5} \mathrm{SUBFP}_{\text {it }}$

$+\quad \mathrm{a}_{6} \mathrm{PFE}_{\mathrm{it}}+\mathrm{a}_{7} \operatorname{Ln}(\mathrm{GLP})_{\mathrm{it}}+$

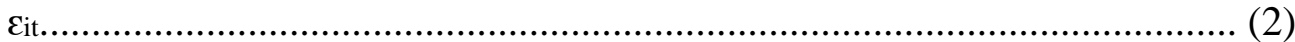

$\mathrm{Au}$ regard de la qualité de nos données et de nos modèles, nous utiliserons les MCG afin de procéder à une régression. Pour le modèle 1 en plus de MCG, il y aura correction des corrélations inter individu entre les erreurs.

\section{Discussions des résultats}

La statistique descriptive

Au regard des statistiques descriptives, on se rend à l'évidence que la dette prise indifféremment du statut légal des institutions de Microfinance est un élément majeur dans leurs financements. On enregistre une moyenne de $66.11 \%$ devant les fonds propres qui ne constituent que $29.41 \%$ dans les ressources des IMFs. En rentrant plus en profondeur, on constate que l'épargne représente $61.11 \%$ de $66.11 \%$ de la dette. Pendant ce temps, les dettes à court et long terme sont autour de $10 \%$.

Quant à la portée sociale, on note une proportion du NE qui va grandissant. Elle enregistre une moyenne de 21305 bien qu'ayant commencé nonchalamment, preuve que cette augmentation date des dernières années. Quant au montant moyen de prêt, on constate que de plus en plus, les IMFs s'écartent des populations cible: on note un minimum de 33958 puis tout à côté, un maximum de 1598 619. Cette tendance qui ramène haut le montant moyen des prêts, mettra à mal les objectifs sociaux des institutions. 


\section{La corrélation}

Il est important de faire un examen des relations univariées entre les variables dépendantes et les variables indépendantes pour déceler s’il existe les problèmes de multi colinéarité. En effet, le Tableau N² présente de façon détaillée les coefficients de corrélation entre les différentes variables.

Tableau N ${ }^{\circ}$. Matrice de corrélation

\begin{tabular}{|c|c|c|c|c|c|c|c|c|c|c|c|}
\hline & ROE & NE & $\operatorname{Ln}(\mathrm{AL})$ & DTA & DLTA & $\begin{array}{l}\text { DC } \\
\text { TA }\end{array}$ & $\begin{array}{c}\text { ET } \\
\text { A }\end{array}$ & $\begin{array}{c}\text { FP } \\
\mathbf{A}\end{array}$ & $\begin{array}{c}\text { SUBF } \\
P\end{array}$ & PFE & $\operatorname{Ln}(\mathbf{G L P})$ \\
\hline ROE & 1.0000 & & & & & & & & & & \\
\hline NE & -0.1080 & 1.0000 & & & & & & & & & \\
\hline $\begin{array}{l}\mathbf{L n}(\mathbf{A L} \\
)\end{array}$ & 0.0876 & -0.1053 & 1.0000 & & & & & & & & \\
\hline DTA & -0.1054 & -0.5339 & -0.0680 & 1.0000 & & & & & & & \\
\hline DLTA & -0.1788 & -0.3292 & $\underline{0.6378}$ & 0.3438 & 1.0000 & & & & & & \\
\hline DCTA & -0.1551 & -0.0813 & 0.1934 & 0.0706 & 0.1242 & 1.0000 & & & & & \\
\hline ETA & -0.0570 & 0.5638 & -0.6037 & $\begin{array}{l}\overline{0} \\
0.0822\end{array}$ & $\underline{-0.7327}$ & $\begin{array}{l} \\
0.1911\end{array}$ & 1.0000 & & & & \\
\hline FPA & 0.1156 & -0.2378 & 0.1997 & 0.0288 & -0.1053 & 0.5115 & 0.0526 & 1.0000 & & & \\
\hline SUBFP & $\underline{0.7593}$ & -0.1800 & 0.1501 & $\begin{array}{l} \\
0.0284\end{array}$ & 0.0332 & 0.0339 & 0.1160 & 0.0499 & 1.0000 & & \\
\hline PFE & 0.0962 & 0.1100 & 0.1091 & $\begin{array}{l}-1216 \\
\end{array}$ & -0.0034 & $\overline{0.0763}$ & 0.0752 & -0.0689 & $\overline{0.0115}$ & 1.000 & \\
\hline $\begin{array}{l}\text { Ln(GL } \\
\text { P) }\end{array}$ & -0.0047 & $\underline{0.7326}$ & 0.2763 & $\begin{array}{l} \\
0.2493\end{array}$ & 0.0731 & $\begin{array}{l} \\
0.1854\end{array}$ & 0.3105 & -0.0885 & $\begin{array}{l} \\
0.1123\end{array}$ & 0.0334 & 1.0000 \\
\hline
\end{tabular}

En analysant le Tableau $\mathrm{N}^{\circ} 2$, on observe bien que très faible, les liaisons négatives entre la dette, la dette à court terme, la dette à long terme, l'épargne sur l'indicateur de profitabilité; ROE. Par contre, on note pour d'autres variables telles que les fonds propres et subventions, une relation positive, tous les liens n'étant pas faibles, car, la subvention a une liaison plutôt importante avec le ROE (75.93\%).

En ce qui Concerne les liaisons entre le NE et la dette, la dette à long terme, la dette à court terme, les fonds propres et les subventions on enregistre des relations négatives. Parmi ces liaisons négatives, on note une qui est importante: celle avec la dette (-53.39\%). Par ailleurs, on remarque une liaison positive et forte avec l'épargne (56.38\%).

Suivant le Ln (AL), on comprend que toutes les liaisons sont positives à l'exception de l'épargne qui est, non seulement négative mais importante ($65.50 \%$ ). Parmi toutes ces liaisons positives, on enregistre celle avec la dette à long terme qui est forte $(75.78 \%)$. En plus de ce qui a été dit sur les corrélations, on constate une relative faiblesse des liens entre les variables indépendantes qui sont plus ou moins inférieures à 50\% à l'exception du lien entre l'épargne et de la dette à long terme qui est de $73.27 \%$. Il est évident que ces deux variables mises ensemble dans une régression est susceptibles de poser un problème de multi colinéarité, d'où la nécessité de leur séparer. 


\section{Analyse de la régression}

Pour régresser les équations de nos modèles, nous avons retenu les moindres carrés généralisés. Spécialement pour les équations $\mathrm{N}^{\circ} 2$ et $\mathrm{N}^{\circ} 3 \mathrm{du}$ modèle $\mathrm{N}^{\circ} 1$ puis $1^{\prime}$ équation $\mathrm{N}^{\circ} 1$ du modèle $\mathrm{N}^{\circ} 2$ on intégrera la correction de la dépendance entre les erreurs. Les régressions sont effectuées sur ces équations afin de définir l'influence qui existe entre les sources de financements et la performance. Comme mesure de la performance, nous utiliserons le ROE, le NEA et le Ln (AL).

Les variables de structure retenues sont entre autres la DTA, DLTA, DCTA, ETA, FPA, SUBFP. A ces variables de structure on associe deux (2) variables de contrôle: PFE et le LnGLP.

Tableau N³. mécanisme de financement et performance mesurée par le ROE

\begin{tabular}{|c|c|c|}
\hline \multirow{2}{*}{ Variables } & \multicolumn{2}{|c|}{ Performance (ROE) } \\
\cline { 2 - 3 } & Modèle 1 & Modèle 2 \\
\hline Constante & -0.5665942 & $-0.1528319^{* * *}$ \\
\hline DTA & 0.0222254 & $-0.885119^{*}$ \\
\hline DLTA & $-0.5451321^{* * *}$ & $-1.127062^{*}$ \\
\hline DCTA & $-0.9555546^{*}$ & $-0.863342^{* *}$ \\
\hline ETA & & $0.2426983^{*}$ \\
\hline FPA & $0.2271611^{* *}$ & $2.485038^{*}$ \\
\hline SUBFP & $2.615423^{*}$ & $0.3069174^{*}$ \\
\hline PFE & 0.3648189 & $0.0076997^{*}$ \\
\hline LnGLP & -0.5665942 & 19.71 \\
\hline R 2 & 0.6834 & 40.26 \\
\hline Wald chi 2 (7) /F $(7,49)$ & 120.89 & 0.0000 \\
\hline Prob $>$ Chi2/ Prob $>$ F & 0.0000 & $10 \%$ \\
\hline
\end{tabular}

$*$ = significativité à $1 \%$; **= significativité à $5 \% ; * * *=$ significativité à $10 \%$

Il découle ce tableau, les analyses suivantes:

- La régression à effet variable de $1^{\prime}$ 'équation $\mathrm{N}^{\circ} 1$ du modèle $\mathrm{N}^{\circ} 1$ et à effet fixe de l'équation $\mathrm{N}^{\circ} 1$ du modèle $\mathrm{N}^{\circ} 2$ nous donne des valeurs de $\mathrm{R}^{2}$ respectivement de $68.34 \%$ et $19.71 \%$. c'est une preuve que les équations sont utilisables bien que la deuxième ayant une faible puissance explicative. Les variables qui affectent positivement la rentabilité des fonds propres dans les IMFs sont: FPA, SUBFP, PFE, LnGLP. De même, ces variables sont toutes significative à un seuil de $5 \%$.

- $\quad$ Il existe aussi un nombre de variables, qui affectent négativement le ROE: il s'agit de DTA, DLTA, DCTA, ETA.

Les variables DTA et DCTA ont une relation négative avec le ROE et significative à 1\%, alors la DLTA est significative à 10\% quand l'épargne est significative à $5 \%$. Ces résultats contredisent les conclusions des travaux de Silva (2008). Ceci peut s'expliquer par le fait que dans la sphère financière camerounaise comme un peu partout en Afrique subsaharienne, il est 
difficile aux banques de financer les PME y compris les IMFs. Pour ce faire, elles durcissent les conditions d'accès aux financements à long terme.

$\underline{\text { Tableau } \mathbf{N}^{\circ} 4}$. structure de financement et performance mesurée par le NEA

\begin{tabular}{|c|c|c|}
\hline \multirow{2}{*}{ Variables } & \multicolumn{2}{|c|}{ Performance (NEA) } \\
\cline { 2 - 3 } & Modèle 1 & Modèle 2 \\
\hline Constante & $-168594.8^{*}$ & $-135575.6^{*}$ \\
\hline DTA & $-17940.71^{*}$ & $-26556.94^{*}$ \\
\hline DLTA & $-49214.82^{*}$ & $/$ \\
\hline DCTA & $43786.39^{*}$ & $43457.03^{*}$ \\
\hline ETA & $/$ & $35300.94^{*}$ \\
\hline FPA & $-15628.14^{*}$ & $-13405.6^{*}$ \\
\hline SUBFP & $-10777.86^{*}$ & $-11353.21^{* *}$ \\
\hline PFE & $8666.981^{*}$ & $11353.21^{* *}$ \\
\hline LnGLP & $9152.04^{*}$ & $6652.104^{*}$ \\
\hline $\mathrm{R}^{2}$ & 0.5681 & 0.7844 \\
\hline Wald chi $2(7) / \mathrm{F}(7,49)$ & 2092.88 & 485.13 \\
\hline Prob $>$ Chi2/ Prob $>$ F & 0.0000 & 0.0000 \\
\hline$*$ significativité à $1 \% ; * *=$ significativité à $5 \% ; * *=$ significativité à $10 \%$
\end{tabular}

Il ressort du Tableau $\mathrm{N}^{\circ} 4$, que les équations $\mathrm{N}^{\circ} 2$ des modèles 1 et 2 ont une grande puissance explicative au regard des valeurs de $\mathrm{R}^{2}$. en plus de cette puissance explicative, s'ajoute la significativité globale des modèles (Prob $>$ Chi2/ Prob $>$ F sont de 0.0000).

- $\quad$ Le signe des variables DTA, DLTA, FPA et SUBFP est négatif. Quant à la significativité, elles sont toutes à 1\%. En utilisant le taux de rotation de la clientèle, Coleman démontre une relation plutôt positive entre la dette et la performance sociale. Quant à la subvention, notre conclusion rejoint celle d'Amendariz et al. (2010) tandis que Bogan et al. (2008) trouvent que la relation est non significative. La raison qui peut être avancée à l'endroit des subventions est que l'incertitude liée à la réception des fonds de subside désorganise l'activité de l'IMF, ce qui n'est pas sans aucune influence sur le nombre d'emprunteurs de l'institution.

- $\quad$ Contrairement à la DTA, DLTA, FPA et SUBFP, les variables telles que la DCTA, l'ETA, le PFE et le LnGLP influencent positivement la variable NEA. En plus, elles sont toutes significative à $1 \%$ à l'exception du PFE qui l'est plutôt à $10 \%$. La justification qui peut être associée à la variable ETA est que dans les IMFs, une importante proportion de l'épargne est détenue par les clients. En effet, les IMFs exigent généralement de leurs clients de constituer auprès d'eux des dépôts. 
Tableau Nº5. Mécanisme de financement et performance mesurée par le LnAL

\begin{tabular}{|c|c|c|}
\hline \multirow[t]{2}{*}{ Variables } & \multicolumn{2}{|c|}{ Performance (LnAL) } \\
\hline & Modèle 1 & Modèle 2 \\
\hline Constante & 10.20332* & 8.996163* \\
\hline DTA & $-0.538889 *$ & 0.0195267 \\
\hline DLTA & $3.419251 *$ & I \\
\hline DCTA & 0.2674412 & 0.3502331 \\
\hline ETA & I & $-2.208256 *$ \\
\hline FPA & $0.4004422 *$ & $0.2746715 * *$ \\
\hline SUBFP & 0.58915* & $\mathbf{0 . 5 3 8 2 3 4 1}$ \\
\hline PFE & 0.5936911* & 0.2775091 \\
\hline LnGLP & 0.1129411* & $0.2360142 *$ \\
\hline $\mathbf{R}^{2}$ & 0.3268 & 0.5503 \\
\hline Wald chi $2(7) / F(7,49)$ & 1082.24 & 126.01 \\
\hline Prob $>$ Chi2 $/$ Prob $>$ F & 0.0000 & 0.0000 \\
\hline
\end{tabular}

Etant donné les $\mathrm{R}^{2}$ (32.68; 55.03), on peut affirmer que les modèles ont une puissance explicative assez considérable. En plus de cette puissance explicative, il est à noter qu'ils sont globalement significatifs à $1 \%$. A l'exception de la DTA et de l'ETA qui ont une influence négative sur les montants moyens de prêts, dont positive sur la profondeur de la portée sociale, les autres à savoir: DLTA, FPA, SUBFP, PFE et LnGLP agissent positivement sur la variable LnAL, dont négative sur la profondeur de la portée sociale.

- Les dettes à long terme permettent de financer les activités aux échéances lointaines. Il est évident que les investissements qui sont de longue durée nécessitent plus de financement que des investissements qui s'étalent sur un temps réduit. La conséquence est que le montant moyen de prêts se verra augmenté par le fait des dettes à long terme.

- $\quad$ Etant donné que dans les IMFs, la plupart de comptes d'épargnes ne sont pas bloqués c'est-à-dire leur propriétaire devrait retrouver leurs fonds en cas de besoin, les IMFs ne pourront s'en servir que dans la mesure où il faut financer les activités de courte durée qui demandent moins de financement.

\section{Recommandations et Conclusion Recommandations}

À la sortie de ces résultats, il est judicieux de formuler un certain nombre de recommandations dans le sens d'améliorer la pérennité des IMFs en impulsant l'efficacité de son action. Pour ce faire, nous recommandons:

Nous recommandons aux IMFs de voir à la hausse leurs montants des dettes à long terme et en même temps, de résoudre l'autonomie de leurs 
activités. Les IMFs devraient aussi consolider l'épargne qu'elles reçoivent et s'assurer de réduire au maximum le niveau de fonds oisifs.

Les IMFs doivent également se rapprocher des populations cible afin de résoudre plus facilement les problèmes liés à l'atteinte des objectifs sociaux.

\section{Conclusion}

L'objectif principal de cette étude est de vérifier si les sources de financement d'une institution de Microfinance influencent aussi bien leurs performances financières que sociale au Cameroun.

Après les régressions suivant la méthode de l'économétrie des données de panels sur un échantillon de huit (8) IMFs camerounaises avec les données recueillies sur une période de huit (8) années de 2006 à 2013, nous avons obtenu plusieurs résultats. Il ressort d'une part que les variables de dettes influencent négativement sur la performance financière et sur la performance sociale. Il s'agit plus spécifiquement de la dette à court terme et de la dette totale. D'autre part que cette même dette totale influence positivement la profondeur de cette même portée. Il représente l'action contraire quant à la dette à long terme. S'agissant de l'épargne, elle affecte négativement la performance financière et positivement la performance sociale. Pour finir, les fonds propres influencent positivement la performance financière et négativement la performance sociale.

Bien que la méthodologie ait tenu en compte la dimension temps et espace, il n'en demeure pas moins que cette étude soit sujette à un certain nombre de limites: Le nombre restreint des variables, liées aussi bien à la performance sociale que la performance financière, retenue dans les modèles de régressions. On aurait pu se servir des variables telles que le rendement de l'actif, auto suffisance opérationnelle, le niveau de pauvreté des clients entre autres.

\section{References:}

1. Abor, J. (2005). The effect of capital structure on profitability: an empirical analysis of listed firms in Ghana, Journal of Risk Finance (Emerald Group Publishing Limited), pp 438-445.

2. Abor, J. (2007). Debt policy and performance of SMEs: evidence from Ghanaian and South Africa firms, Journal of Risk Finance, Vol. 8 pp.364-79.

3. Adair, P. \& Berguiga, I. (2010). «Les facteurs déterminants de la performance sociale et de la performance financière des institutions de microfinance dans la région MENA: une analyse en coupe instantanée». Région et Développement, vol. 32, pp. 91-120. 
4. Akerlof, G. A. (1970). «The Market for "Lemons": Quality Uncertainty and the Market Mechanism ». The Quarterly Journal of Economics, vol. 84, N³, pp. 488-500.

5. Albouy, M. et al. (2003), «les grands auteurs en finance». Ed. EMS Management.

6. Armendariz, B. \& Morduch, J. (2010). The economics of microfinance. Cambridge.

7. Ayayi, A. \& Sene, M. (2010). «What Drives Microfinance Institution Financial Sustainability». The Journal of Developing Areas, vol. 44, $\mathrm{N}^{\circ} 1$, pp. 303-322

8. Ayayi, A. G. (2012). Microfinance: A Time to Deliberate. Asian Economic and Financial. Review, 2(3), 445-447.

9. Berger, A. N. \& Bonaccorsi Di Patti, E. (2006). Capital Structure and Firm Performance: A New Approach to Testing Agency Theory and an Application to the Banking Industry, Journal of Banking and Finance, Vol. 30, pp. 1065-1102.

10. Bogan, V. (2008). Microfinance Institutions: Does Capital Structure Matter?, Department of Applied Economics and Management, Cornell University, 454 Warren Hall, Ithaca, NY 14853. Retrieved from.

11. Bogan, V., Johnson, W. \& Mhlanga, N. (2008). Does Capital Structure Affect the Financial Sustainability of Microfinance Institutions?, Department of Applied Economics and Management, Cornell University, 454 Warren Hall, Ithaca, NY 14853.

12. Bogan, V., Johnson W \& Mhlanga, N. (2007). Does Capital Structure Affect the Financial Sustainability of Microfinance Institutions?, Department of Applied Economics and Management, Cornell University, 454 Warren Hall, Ithaca, NY.

13. Caroll, A. B. (1979). A three dimensional conceptual model of corporate social performance, Academy of Management Review, 4(4), pp. 497-505.

14. Caudill, S. D., Gropper \& Hartarska, V. (2009). "Which microfinance institutions are becoming more cost-effective with time?, Evidence from a mixture model," Journal of Money, Credit, and Banking, 41, 651-672.

15. CGAP (2003). Consensus-Guidelines-Definitions-of-SelectedFinancial-Terms-Ratios-and-Adjustments-for-Microfinance-French (1).

16. CGAP (2007). MFI Capital Structure Decision Making: A Call for Greater Awareness. 
17. Crombrugghe et al. (2008). Grafting and characterization of protein on polyaniline surface for biosensor applications, Volume 40, Issue 3-4, pp. 404-407.

18. Hartarska, V. \& Nadolnyak, D. (2008). «Does rating help microfinance institutions raise funds? Cross-country evidence», International Review of Economics and Finance, vol. 17, $\mathrm{N}^{\circ} 4$, pp. 558-571.

19. Hartarska, V. (2005). «Governance and Performance of Microfinance Institutions in Central and Eastern Europe and the Newly Independent States». World Development, vol. 33, $\mathrm{N}^{\circ} 10$, 1627-1643.

20. Haruna Sekabira (2013). Capital Structure and Its Role on Performance of Microfinance Institutions: The Ugandan Case. Sustainable Agriculture Research; Vol. 2, No. 3.

21. Hudon, M. \& Traca, D. (2011). On the efficiency effects of subsidies microfinance: An empirical inquiry, World Development, 39, 966-973.

22. Jensen, M. C. (1976). Agency Costs of Free Cash Flow, Corporate Finance, and Takeovers, American Economic review, 76, pp 323339.

23. Jensen, M. \& Meckling, W. (1976). Theory of the Firm: Managerial Behavior, Agency Costs and Ownership Structure, Journal of Financial Economics, 3: 305-360.

24. Kar, A. K. (2012). Does capital and financing structure have any relevance to the performance of microfinance institutions?, International review of applied economics.

25. Kochhar (1996). Institutional Investors and Firm Innovation: a Test of Competing Hypotheses, Full publication history. PP. 23-57.

26. Kyereboah-Coleman, A. (2007). The impact of capital structure on the performance of microfinance institutions. [Article]. Journal of Risk Finance (Emerald Group Publishing Limited), 8(1), 56-71.

27. Labie M. (1999). «La microfinance en questions: Limites et choix organisationnels», Bruxelles, Editions Luc Pire.

28. Lapenu, C. \& Doligez, F. (2007). «Mesure de la performance sociale: les implications pour le secteur de la microfinance», Revue internationale de l'économie.

29. Littlefield, E. \& Rosenberg, R. (2008). «La microfinance et les pauvres, la démarcation entre microfinance et le secteur financier s'estompe», Technique financière de développement, $\mathrm{N}^{\circ} 78$.

30. Luzzi, F. G. \& Weber, S. (2006). Measuring the performance of microfinance institutions, Microfinance and Public Policy, pp 153169. 
31. Mc Kinnon, R.I. (1973). Money and Capital in Economic Development. Washington DC, Brookings Institution.

32. Mersland, R. \& Strom, R. (2009). Performance and governance in microfinance institutions. [Article], Journal of Banking and Finance, N³3, pp. 662-669.

33. Modigliani, F. \& Miller, M. H. (1963). «Corporate Income Taxes and the Cost of Capital: A Correction». American Economies Review, Vol.53, N³, (June), p. 433-443.

34. Modigliani, F. \& Miller (1958). The cost of capital, corporate finance and the theory of investment", American Economic Review.

35. Myers, S. \& Majluf, N.S. (1984). Corporate financing and investment decisions when firms have information that investors do not have, Journal of Finance, vol. 39 (June), 187-221.

36. Nawaz (2010). Issues in Subsidies and Sustainability of Microfinance: An Empirical Investigation, Université Libre de Bruxelles-Solvay Brussels School of Economics and Management Centre Emile Bernheim.

37. Philippe, A. \& Imene, B. (2010). «Les facteurs déterminants de la performance sociale et de la performance financière des institutions de microfinance dans la région MENA: une analyse en coupe instantanée», Région et Développement N³2, pp. 15-30.

38. Robinson (1997). Meiotic Origin of Trisomy in Confined Placental Mosaicism is Correlated with Presence of Fetal Uniparental Disomy, High Levels of Trisomy in Trophoblast, and Increased Risk of Fetal Intrauterine Growth Restriction. Am J Hum Genet. vol, 60(4), PP. 917-927.

39. Sanford, J., Grossman \& Oliver D. Hart (1982). Corporate Financial Structure sand Managerial Incentives; The Economics of Information and Uncertainty, Volume Author/Editor: John J. McCall, ed., Volume Publisher: University of Chicago Press, Volume ISBN: 0-226-55559-3, (p. 107-140).

40. Servet (2007). «Jean-Michel, Banquiers aux pieds nus. La microfinance», Les Cahiers d'Outre-Mer, 238, pp. 275-277.

41. Shaw Edward (1973). Financial Deepening in Economic Development, New York: Oxford University Press.

42. Silva, M. W. S. S. (2008). The effect of capital structure on microfinance institutions performance, Master's thesis, University of Agder, Kristiansand.

43. Simerly, Roy, L. \& Mingfang, LI. (2000). Market Dynamism, Capital structure and performance: A theoretical integration and an empirical test, Strategic Management Journal, vol. 21, 31-49. 
44. Stiglitz (1976). Equilibrium in Competitive Insurance Markets: An Essay on the Economics of Imperfect Information, The Quarterly Journal of Economics, vol. 90, issue 4, pp. 629-649.

45. Stiglitz, J. E (1969). A Re-Examination of the Modigliani-Miller Theorem, American Economic Review, vol 59:5, PP. 784-793.

46. Strong Vi Ngo (2012). Capital structure and Microfinance performance, a cross country analysis and case study of Vietnam, $\mathrm{PhD}$ thesis, University of Birmingham.

47. Vernimmen, P. (2002). «Finance d'entreprise», Brocher. 